\title{
Limits on Astrometric Accuracy
}

\author{
Alan L. Fey \\ United States Naval Observatory
}

\begin{abstract}
A general overview of the various factors limiting the accuracy to which astrometric observations can be made is presented. Since this is a very broad topic, it is limited primarily to radio astrometry of extragalactic objects.
\end{abstract}

\section{VLBI astrometry - a brief review}

Wide-angle astrometry at radio frequencies is made possible by the technique of Very Long Baseline Interferometry (VLBI). The basic VLBI observable for astrometry is the group delay, which is related to the difference in the time of arrival of radio signals from a distant source at pairs of radio telescopes. Astrometric VLBI observations are made at standard frequencies of $2.3 \mathrm{GHz}$ and $8.4 \mathrm{GHz}$. The radio telescopes can be separated by distances up to the diameter of the Earth and can measure the group delay with a precision on the order of $10 \mathrm{ps}(\approx 3 \mathrm{~mm}$ or $60 \mu$ as on a $10000 \mathrm{~km}$ baseline $)$. Angular resolution on the sky on the order of 1 milliarcsecond ( 1 mas $\approx 4 \mathrm{pc}$ at $z=1$ ) is routinely achieved for imaging observations. The objects observed for astrometry are usually quasars and active galactic nuclei, which are assumed to be at great distances.

\section{Basic aspects of VLBI astrometry}

In the absence of errors, a measurement of group delay yields the geometric delay, which determines the angle between the baseline and the source position vectors (the baseline is a vector connecting two radio telescopes and the source vector points to the position of the radio source on the sky). By knowing the position of the antennas on the surface of the Earth and the orientation of the Earth in space, the position of the source on the sky can be readily computed. In practice, the group delay measured by a VLBI interferometer is contaminated by various additional unwanted delays which must be corrected in order to obtain accurate source positions. These additional delays can be grouped into three categories: 1) delays introduced by the radio source itself, 2) delays introduced by an intervening medium, and 3 ) delays introduced by the observing system. These additional delays are discussed here only briefly. A more detailed discussion can be found in the review by Sovers, Fanselow, \& Jacobs (1998). 


\subsection{Source}

Extragalactic radio sources are assumed to be very distant (typical redshifts of about 1.0) and thus should exhibit little or no detectable proper motions. However, most of the compact extragalactic sources which are used for astrometry have variable emission structure on scales larger than the accuracy of their position estimates. Fey \& Charlot $(1997,2000)$ have shown that intrinsic structure contributions to the measured group delay are significant, ranging from maximum delay corrections of only a few picoseconds for the most compact sources to maximum delay contributions of several nanoseconds for the most extended sources. These affects are variable and mostly unpredictable.

\subsection{Intervening medium}

Before reaching an observer on Earth, the cosmic signals from extragalactic objects must pass through the intergalactic, interstellar, interplanetary, and terrestrial media. Both the charged and neutral components of these media modify the propagation speed of the signals. Anisotropies in the media along different ray paths can distort the wavefront and introduce a differential delay in the arrival time of the signals (with respect to propagation in a vacuum) at two widely separated radio telescopes. These path length differences become significant only in the vicinity of the Earth. The most important contributions come from the troposphere and the ionosphere where the maximum delay contributions are on the order of $100 \mathrm{~ns}$ and $10 \mathrm{~ns}$, respectively. These affects are variable but can be modeled and virtually eliminated. Tropospheric delays can be modeled to about the $10-50 \mathrm{ps}$ level. The dispersive delay contribution from the ionosphere can be corrected to better than a few picoseconds using dual-frequency observations.

\subsection{Observing system}

The observing system consists of the radio telescopes (including their structure and instrumentation), the baseline geometry, the positions of the radio telescopes on the surface of the Earth, and the orientation of the Earth in three-dimensional space. Antenna structure and instrumentation can contribute to the measured delay with maximum contributions on the order of $100 \mathrm{~ns}$ and $1 \mu \mathrm{s}$, respectively. The baseline geometry is dominated by the purely geometric component which can contribute as much as $20 \mathrm{~ms}$. Abberation due to the Earth's motion can contribute as much as $2 \mu$ s and the gravitational delay due to the Sun can be on the order of $10 \mathrm{~ns}$ for observations at about $10^{\circ}$ from the Sun. The positions of the radio telescopes are affected by tectonic and tidal motions, which can contribute at the level of several nanoseconds. Earth orientation contributes to the measured delay at about the $100 \mathrm{~ns}$ level for UT1/polar motion and at about the $1 \mu \mathrm{s}$ level for nutation and precession. These affects are variable and mostly predictable and can be modeled at the several tens of picosecond level.

\section{Compact extragalactic radio sources}

\subsection{Observation}

The compact extragalactic objects commonly observed for astrometry are usually extended on milliarcsecond scales. Many of the observed sources have dom- 


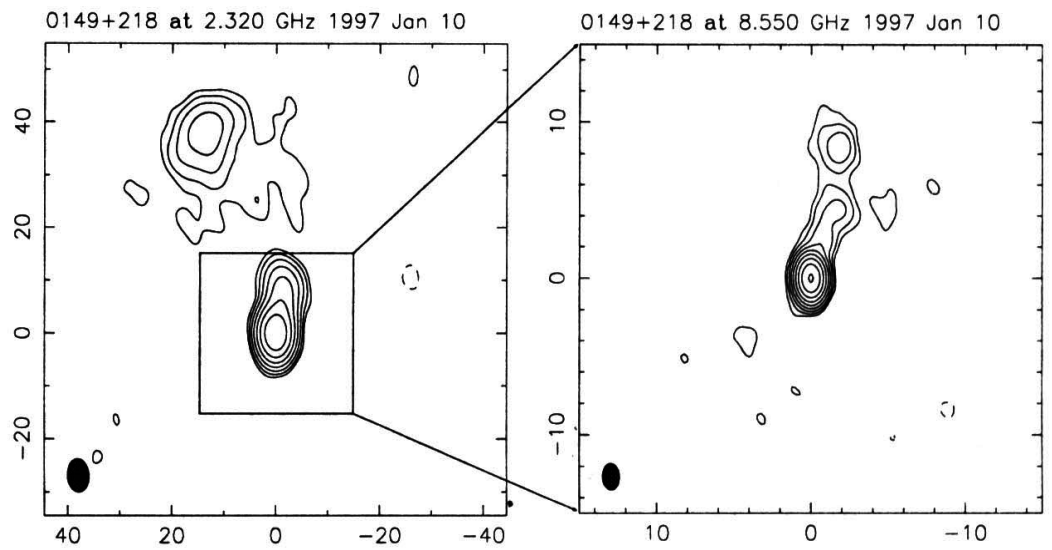

Figure 1. Contour plots of the radio emission from the source $0149+218$. The left panel shows the radio emission at a frequency of $2.3 \mathrm{GHz}$ while the right panel shows the emission at $8.4 \mathrm{GHz}$.

inant cores containing the majority of the total flux density and angular sizes less than several milliarcseconds. However, at some level, almost all of the observed sources show structure characteristic of a one-sided core-jet morphology, often with multiple jet components. An example core-jet radio source is shown in Figure 1. The jet components in core dominated sources often show motion with an apparent transverse velocity, $\beta_{\text {app }}=\frac{v_{\text {app }}}{c}$, greater than the speed of light. Motion with $\beta_{\mathrm{app}}>c$ is commonly referred to as superluminal motion. A wide range of $\beta_{\text {app }}$ have been observed in different sources. Different $\beta_{\text {app }}$ for different jet components in the same source have also been observed. Jet components can accelerate, split or even merge. Stationary components can also coexist with moving components.

\subsection{Theory}

In the standard theory of extragalactic radio sources (Blandford and Königl 1979), emission from quasars and active galactic nuclei is assumed to be powered by a central engine (presumably a black hole) where energetic phenomena occur. The radio jets are modeled as well-collimated radiating plasma flows moving at relativistic velocity away from the central engine. Opacity effects in these sources contribute to variations in the observed emission structures as a function of frequency. In particular, opacity changes in the core regions will produce frequency dependent changes in the position of the peak emission of the core, presumably along the position angle of the jet emission. In this model, the core is the base of the jet where the optical depth is approximately unity. The position of the core should appear to be further downstream at lower frequencies. The apparent component motions that we measure are probably phase velocities of a moving disturbance in the jet, such as a shock, and may not be simply related to the velocity of the underlying plasma flow. A schematic representation of a core-jet radio source is shown in Figure 2.

The observed transverse velocity of separation of a component from the core, $v_{\text {app }}=\beta_{\text {app }} c$, is related to the true velocity, $v=\beta c$, and the angle to the 


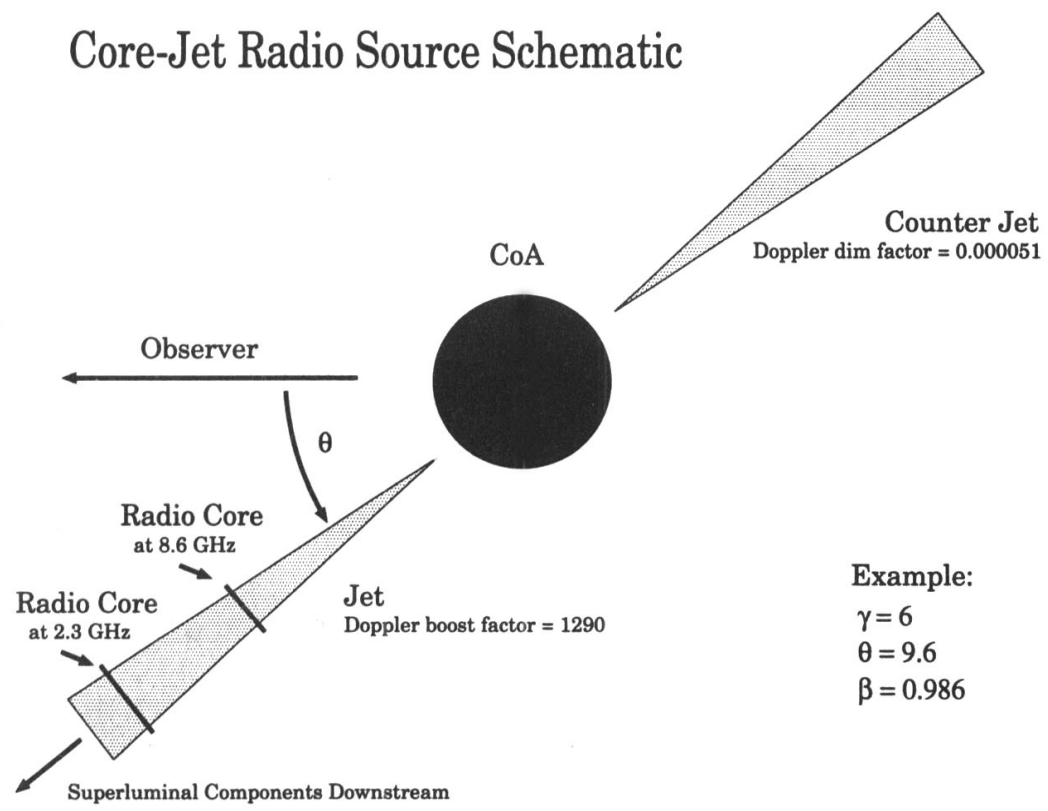

Figure 2. A schematic representation of a core-jet radio source. The Center of Activity (CoA) is presumably a massive black hole. The example Doppler boosting (dimming) factor is given for a Lorentz factor, $\gamma=6$, an angle between the jet direction and observer, $\theta=9^{\circ} .6\left(170^{\circ} 4\right)$, a jet velocity, $\beta=\frac{v}{c}=0.986$, and a power-law flux density spectral index, $\alpha=-1$. 
line of sight, $\theta$, by

$$
\beta_{\text {app }}=\frac{\beta \sin \theta}{1-\beta \cos \theta},
$$

where $\theta=0^{\circ}$ corresponds to motion directly toward the observer and $\theta=90^{\circ}$ corresponds to motion transverse to the line of sight.

The apparent surface brightness of the components will be affected by relativistic aberration. For the simple case of an optically thin plasma "blob" moving at angle, $\theta$, towards the observer with velocity, $v=\beta c$, emitting isotropic flux density, $S_{0}(\nu)$ at frequency $\nu$, the observer will measure a flux density

$$
S(\nu)=S_{0}\left(\frac{\nu}{\delta}\right) \delta^{3}=S_{0}(\nu) \delta^{3-\alpha},
$$

where the last equality holds for the case of a source with a power-law flux density spectrum, $S(\nu) \propto \nu^{\alpha}$, and

$$
\delta=\gamma^{-1}(1-\beta \cos \theta)^{-1}
$$

is the Doppler factor. Radiation emitted from the approaching jet is Doppler boosted while radiation emitted from the receding jet is Doppler dimmed. The result is the one-sided core-jet morphology commonly observed. For a more thorough review of superluminal radio sources, see Pearson \& Zensus (1987) and references therein.

\subsection{Structure monitoring}

Maintenance of the International Celestial Reference Frame (ICRF) at a high level of accuracy requires measuring and monitoring changes in source structure in order to determine both the short and the long term effects of variable intrinsic source structure on astrometric position determination. To this end, the U.S. Naval Observatory has initiated and is continuing an observing program to image the radio reference frame sources on a regular basis at the same radio frequencies used for precise astrometry ${ }^{1}$.

\section{Structure effects on astrometric positions}

\subsection{Superluminal motion of jet components}

As mentioned previously, the compact extragalactic sources used for astrometry have variable and unpredictable emission structure which can, in some cases, induce temporal variations of their derived astrometric positions. Fey, Eubanks, \& Kingham (1997) report that the radio source 0923+392 exhibited significant proper motion during the period 1986-1997 as observed by astrometric and geodetic VLBI. The observed astrometric proper motion for $0923+392$ agrees well with the superluminal motion of the brightest component found in VLBI

\footnotetext{
${ }^{1}$ Data obtained to date are available for scientific use by anyone and can be accessed from the United States Naval Observatory's Radio Reference Frame Image Database at http://wrv.usno.navy.mil/RRFID/.
} 


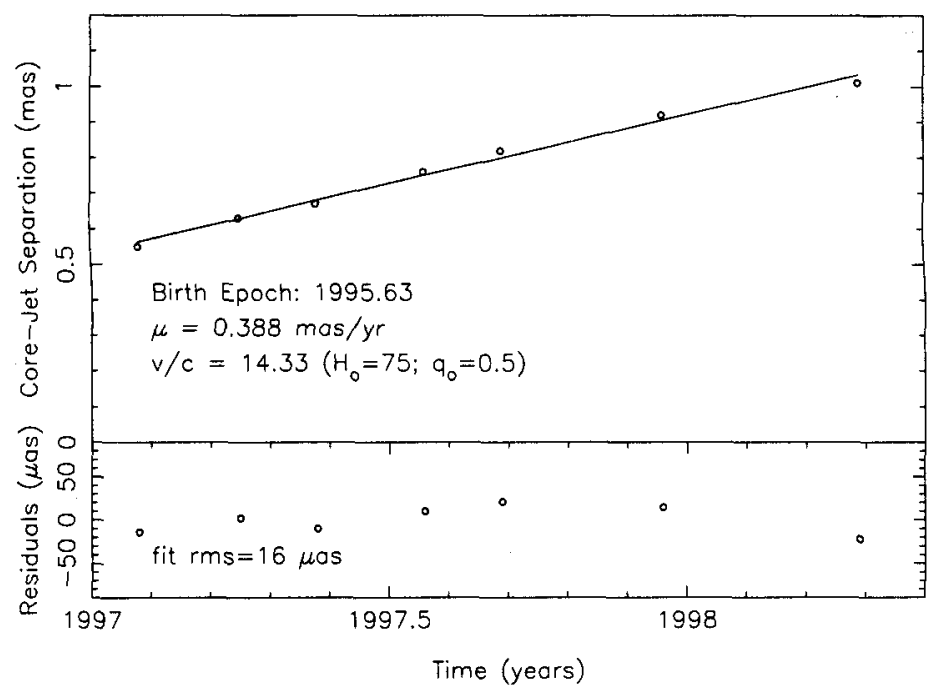

Figure 3. The time variability of the angular separation between the core and a superluminal component in the radio source $1308+326$ at a frequency of $8.4 \mathrm{GHz}$. The superluminal component has a fitted separation rate of $0.388 \mathrm{mas} / \mathrm{yr}$, which translates into a $\beta_{\text {app }}=14.33$ $\left(z=0.996 ; \mathrm{H}_{0}=75 \mathrm{~km} \mathrm{~s}^{-1} \mathrm{Mpc}^{-1}, \mathrm{q}_{0}=0.5\right)$. "The extrapolated component birth epoch is 1995.63 , assuming no acceleration.

images. The time variability of the angular separation between the core and a superluminal component in the radio source $1308+326$ is shown in Figure 3. These results were obtained by fitting Gaussian models to the observed brightness distribution of the source in $8.4 \mathrm{GHz}$ images obtained at seven different epochs between 1997 and 1999.

The effect of the time variability of the intrinsic structure of $1308+326$ on the measured astrometric position is shown in Figure 4 and Figure 5. As can be seen in these figures, the changing intrinsic structure induces significant position variations of up to several tenths of a milliarcsecond in each coordinate. Also note that significant position deviations began c. 1994, almost one and one half years before the extrapolated birth epoch of the superluminal component (c. 1995.63), suggesting that the superluminal component has accelerated since its birth.

\subsection{Opacity effects in the core}

Opacity changes in the core regions of extragalactic sources may produce frequency dependent changes in the position of the peak emission of the core. In the standard relativistic jet model previously discussed (Blandford and Königl, 1979), the core is the base of the jet where the optical depth is approximately unity. The position of the core should appear to be further downstream at lower frequencies (as depicted in Figure 2). This has been observed for the case of 


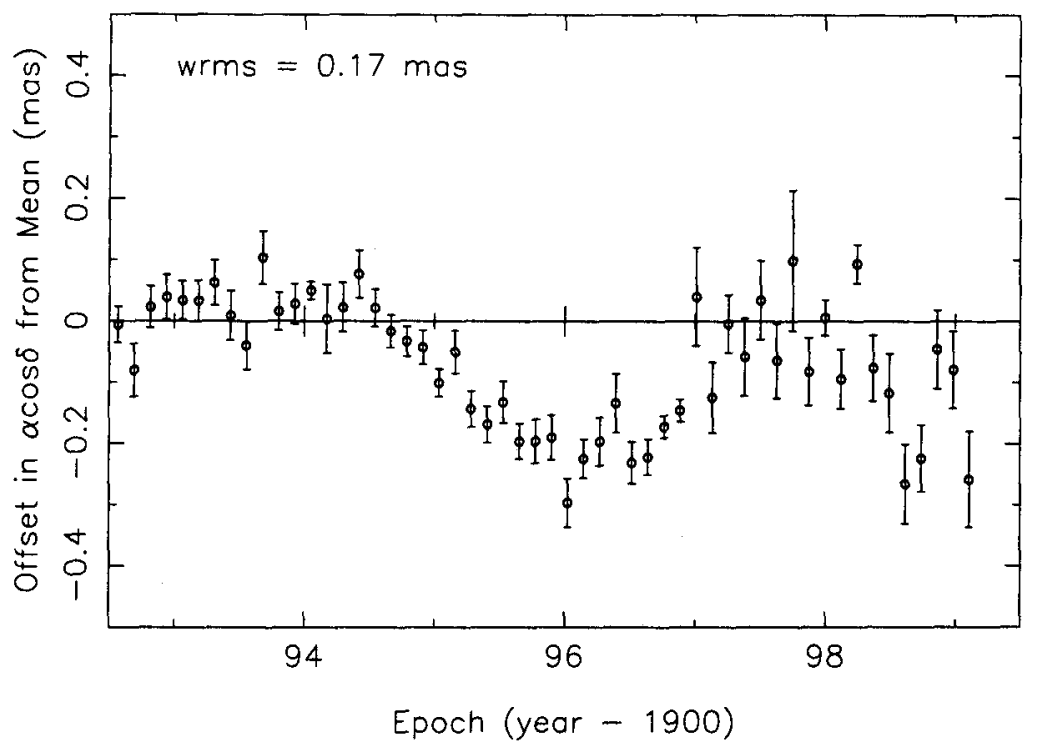

Figure 4. The astrometric position time series for $1308+326$ show as a relative offset in right ascension, $\alpha \cos \delta$, from an absolute as. trometric position. The weighted root-mean-square (wrms) about the mean position is also listed.

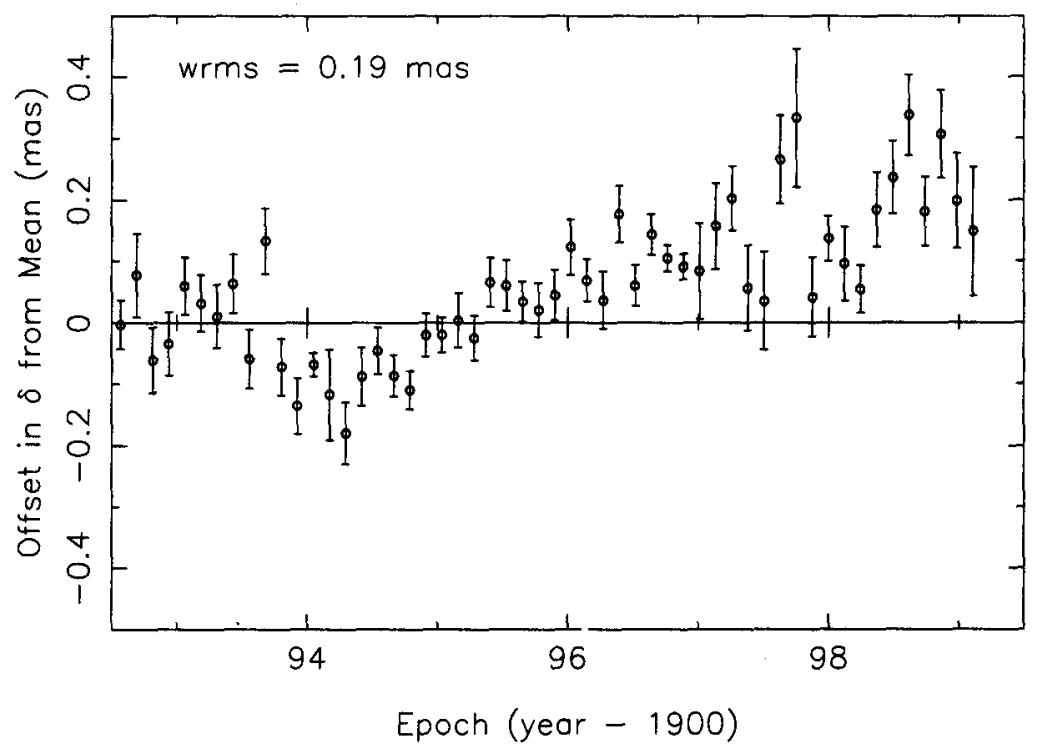

Figure 5. The astrometric position time series for $1308+326$ shown as a relative offset in declination, $\delta$, from an absolute astrometric position. The weighted root-mean-square (wrms) about the mean position is also listed. 
the quasars $1038+528$ A \& B. From phase-referenced observations, Rioja et al. (1997) found a frequency dependence of the position of the core of $\mathrm{A}$ with respect to $B$ of the order 0.7 mas between $2.3 \mathrm{GHz}$ and $8.4 \mathrm{GHz}$ with the smaller separation being at $2.3 \mathrm{GHz}$.

\section{Other related effects on astrometric positions}

\subsection{The ionosphere and dispersive media}

The extra delay introduced by the Earth's ionosphere is dispersive and is proportional to the inverse square of the observing frequency (in the limit that the observing frequency is much greater that the plasma frequency, which is a very good approximation for the ionosphere, and in the absence of magnetic fields).

Dual frequency observations at two widely separated frequencies can be employed to remove the ionospheric delay. If delay observations $\tau_{1}$ and $\tau_{2}$ are made at sky frequencies $\nu_{1}$ and $\nu_{2}$, then the ratio

$$
\frac{\tau_{1}}{\tau_{2}}=\left(\frac{\nu_{2}}{\nu_{1}}\right)^{2}
$$

can be used to calculate the free-space delay. As mentioned previously, this is standard procedure for most astrometric VLBI observations.

The intergalactic, interstellar, and interplanetary media will also contribute to the measured delay but, since the dispersive nature of the ionized component of these media is, to first order, the same as that of the Earth's ionosphere, these effects will be removed at the same time.

\subsection{Angular broadening}

Turbulent electron density fluctuations in the interstellar medium are revealed by both diffractive and refractive scattering of radio waves from Galactic and extragalactic sources. One manifestation of diffractive scattering is the angular broadening of compact sources to almost Gaussian brightness distributions (Rickett, 1990).

Although the dominant contribution for lines of sight within about $10^{\circ}$ of the galactic plane is from the interstellar medium, for lines of sight close to the Sun, the interplanetary medium will dominate. Contributions from the intergalactic medium have not been detected by angular broadening. A broadened source will have a measured angular size that scales with frequency as

$$
\theta_{\text {scattered }} \propto \nu^{-2.2},
$$

for an assumed Kolmogorov spectrum of turbulent electron density fluctuations.

Standard VLBI astrometric observations are made at frequencies of $2.3 \mathrm{GHz}$ and $8.4 \mathrm{GHz}$. As sources are observed closer to the galactic plane (or the Sun), source size will increase (faster at $2.3 \mathrm{GHz}$ than at $8.4 \mathrm{GHz}$ ). This will have the effect that observation signal-to-noise-ratio (SNR) will decrease as a consequence of decreased correlated flux density, especially on longer baselines. Along heavily scattered lines of sight, a source can become completely resolved at $2.3 \mathrm{GHz}$ (no detection), in which case no ionosphere dispersion correction can be applied, making the data almost useless. The deficit of ICRF sources in the galactic plane is a direct result of angular broadening. 


\section{Summary}

Intrinsic structure in the extragalactic objects used for VLBI radio astrometry contributes to the measured delay at the few picoseconds level for the most compact sources but can be up to several nanoseconds for the most extended sources. These affects are variable and mostly unpredictable and, if uncorrected, will limit VLBI accuracies for individual sources to the several hundred microarcsecond level. Structure monitoring is currently underway with the hope that when sources become unsuitable for continued use they can be eliminated from astrometric solutions or that structure corrections can be applied to the astrometric data to improve the solutions. The additional delays introduced by the Earth's atmosphere are variable but can be modeled and virtually eliminated. Tropospheric delays can be modeled to about the $10-50 \mathrm{ps}$ level. Together with source structure, uncertainties in modeling the water vapor content of the troposphere are the two major sources of error in astrometric VLBI. The dispersive delay contribution from the ionosphere can be corrected to better than a few picoseconds using dual-frequency observations. The contribution of the observing system to observed delays can be modeled at the several tens of picosecond level. Increasing the accuracy of VLBI astrometry to the microarcsecond level will require improved models for source structure and the troposphere as well as improved models for VLBI antenna structure, instrumentation, and positions.

\section{References}

Blandford, R. D. \& Königl, A., 1979, Astrophys. J., 232, 34.

Fey, A. L. \& Charlot, P., 1997, Astrophys. J. Sup., 111, 95.

Fey, A. L. \& Charlot, P., 2000, Astrophys. J. Sup., in press.

Fey, A. L., Eubanks, T. M., \& Kingham, K. A., 1997, Astron. J., 114, 2284.

Pearson, T. J. \& Zensus, J. A., 1987, in Superluminal Radio Sources, Proceedings of a Workshop in honor of Professor Marshall H. Cohen, held at Big Bear Solar Observatory, California (October 28-30, 1986), J. Anton Zensus \& Timothy J. Pearson (eds), Cambridge University Press, 1.

Rickett, B. J., 1990, ARAEBA, 28, 561.

Rioja, M. J., Marcaide, J. M., Elósegui, P., \& Shapiro, I. I., 1997, Astron. Astrophys., 325, 383.

Sovers, O., Fanselow, J., \& Jacobs, C., 1998, Reviews of Modern Physics, Vol. 70, 1393. 